\title{
An FPGA-based lock-in detection system to enable Chemical Species Tomography using TDLAS
}

\author{
Andrea Chighine ${ }^{1}$,Edward Fisher ${ }^{1}$, David Wilson ${ }^{2}$, Michael Lengden ${ }^{2}$,Walter Johnstone ${ }^{2}$ and Hugh McCann ${ }^{1}$ \\ ${ }^{1}$ School of Engineering, The University of Edinburgh, Edinburgh, Scotland, UK \\ ${ }^{2}$ Department of Electronics and Electrical Engineering, University of Strathclyde, Glasgow, Scotland, UK
}

\begin{abstract}
This paper presents the design, implementation and test of a compact, low-cost and fully digital signal recovery system for tunable diode laser absorption spectroscopy (TDLAS) in narrow line-width gas sensing applications. An FPGA-based digital lock-in amplifier (DLIA), in conjunction with TDLAS using the wavelength modulation spectroscopy (WMS) technique, is utilized to demodulate and extract first (1f) and second (2f) harmonic signals for a narrow $\mathrm{CO}_{2}$ feature in the spectrum region of $1997.2 \mathrm{~nm}$. The spectrum in this wavelength region shows suitably weak water absorption, enabling $\mathrm{CO}_{2}$ detection with high resolution. Gas-cell experiments were carried out using the DLIA and a conventional rack-mounted commercial lock-in amplifier. The comparison between the two systems shows good agreement, validating the feasibility of this approach and demonstrating the prospect for extension to a massively multichannel system to implement Chemical Species Tomography.
\end{abstract}

Keywords-Field Programmable Gate Arrays (FPGA), Lock-in amplifier, Digital demodulation, Tunable Diode Laser Spectroscopy (TDLAS), Chemical Species Tomography (CST)

\section{INTRODUCTION}

Over the last two decades, tunable diode laser-absorption spectroscopy (TDLAS) has become established as a robust and widely utilized technique for sensing and measuring gas parameters in a variety of applications [1]. Over the same period, high-speed Chemical Species Tomography (CST) has become established [2], using predominantly fixedwavelength diode-laser technology.

Using continued development of narrow line-width laser technologies, TDLAS has evolved into a highly selective spectroscopic technique, which can detect the unique absorption fingerprints of various gases. Light emitted from a tunable diode laser is passed through a gas sample to a photodiode, and the detectable absorption of light can be related to the gas properties, such as concentration, pressure, and temperature. This optical absorption modality is widely utilized, as being non intrusive, fast and sensitive. For gas detection in harsh, industrial environments and at low concentration, few methods have been proposed to improve upon the detection sensitivity of TDLAS [3]. A particular method which greatly reduces the influence of laser and electronic noise and is well suited to targeting gas species with sharp absorption features, is wavelength modulation spectroscopy (WMS). The basic principle of WMS is to simultaneously modulate the laser wavelength with a sinusoidal high frequency signal ( $f$, in the order of tens of $\mathrm{kHz}$ ) while scanning the laser wavelength across the absorption profile of interest at low frequency (in the order of tens of $\mathrm{Hz}$ ). The interaction between the modulated laser signal and the non-uniform absorption profile gives rise to several components in the frequency domain, which can be related to the harmonics of the laser modulation signal [4]. Lock-in amplification, using phase sensitive detection (PSD), is capable of detecting, selecting and isolating these harmonic components. By implementing a narrow band-pass filter, through heterodyne mixing electronics, the $1 \mathrm{f}$ and $2 \mathrm{f}$ harmonic signals can be detected, thus obtaining absorption information.

Although important improvements in noise suppression are achieved by applying the WMS method, the harmonic components detected by the photodiode, before the lock-in stage, are not easy to analyze because of the low level gas signal being affected by noise [5]. Lock-in amplifiers, commonly used in instrumentation for their capability of detecting low-level signals affected by interference and noise, are therefore fundamental to the processing of gas signals in the TDLAS technique [6]. Continued development of Field Programmable Gate Array (FPGA) technology has allowed the implementation of low-cost and compact complex systems, while obtaining both high reliability and, in instrumentation systems, high accuracy. The progress in these technologies has prompted a number of implementations of digital lock-in amplifiers (DLIA) on FPGA platforms [7-8].

This paper demonstrates a fully digital $\mathrm{CO}_{2}$ TDLAS system with the FPGA-based lock-in amplifier technique using phase sensitive detection. The implementation is built using a mid-level Xilinx Spartan6 FPGA with an analog to digital converter (ADC), processing the wavelengthmodulated signal emitted by a diode laser tuned to scan the $\mathrm{CO}_{2}$ absorption feature at $1997.2 \mathrm{~nm}$ [9]. The system discussed here is to be implemented subsequently for a 126beam CST system for $\mathrm{CO}_{2}$ imaging in a gas turbine exhaust plume [10-11].

The fundamentals of TDLAS and the lock-in measurement principle are described in the next section. Section III discusses the implementation and experimental setup and section IV presents preliminary measurement results comparing the FPGA-based DLIA and a conventional lock-in amplifier instrument. 


\section{METHODS}

\section{A. Fundamentals of TDLAS}

The fundamental theory governing absorption spectroscopy is given by the Beer-Lambert law (eq. 1). The ratio of the transmitted light intensity $I_{t}$ through an absorbing medium and the incident laser radiation $I_{0}$, at frequency $v$, is exponentially proportional to the total path length $L$, the total pressure $P$, the temperature $T$, the line strength of the molecular energy transition $S$, the mole fraction of the absorbing species $x$ and the line shape function $\phi$ :

$$
\left(\frac{I_{t}}{I_{0}}\right)_{V}=e^{-S[T] \phi P x L}
$$

and the absorbance $\alpha_{v}$ is then defined as follows:

$$
\alpha_{v}=-\ln \left(\frac{I_{t}}{I_{0}}\right)_{v}=-S[T] \phi P x L
$$

\section{B. WMS Principles}

Fig. 1 shows the WMS scanning principle. The diode laser wavelength is scanned across the gas absorption feature by drive-current modulation with a repetitive sawtooth waveform. An additional high frequency modulation is superimposed on the sawtooth waveform. This sinusoidal modulation, which can be in the range from tens of $\mathrm{kHz}$ to several $\mathrm{MHz}$, generates an additional modulation at frequency $\mathrm{f}$ in both laser intensity and wavelength. The laser wavelength dithers across the absorption feature, interacts with the absorption line and generates several harmonic components in the photo-detected signal. The latter is processed by the lock-in amplifier, which has capabilities to isolate specific harmonic components. In WMS, measurements mainly consider the first (1f) and second (2f) harmonics, which are obtained by the lock-in amplifier multiplying the photo-detected signal by a reference signal at the frequency of interest. This operation brings the desired signal to d.c., and spurious frequency components can be easily removed by filtering. Fig. 2 shows the wavelength and intensity modulation of the laser with the absorption feature, which gives rise to the $1 \mathrm{f}$ signal, proportional to the first derivative of the absorption feature $[4,5]$.

Starting from the Beer-Lambert law approximated for small absorption $\left(\alpha_{v}<<1\right)$

$$
I_{t}=I_{0} e^{-\alpha} \approx I_{0}[1-\alpha]
$$

then applying a sine dither signal superimposed to the ramp waveform as follows (with $\omega=2 \pi f$ ),

$$
\Delta I \cos (\omega t) \rightarrow\left[I_{0}+\Delta I \cos (\omega t)\right][1-\alpha C l]
$$

and using the Taylor series approximation for the absorption function to second order, we obtain:

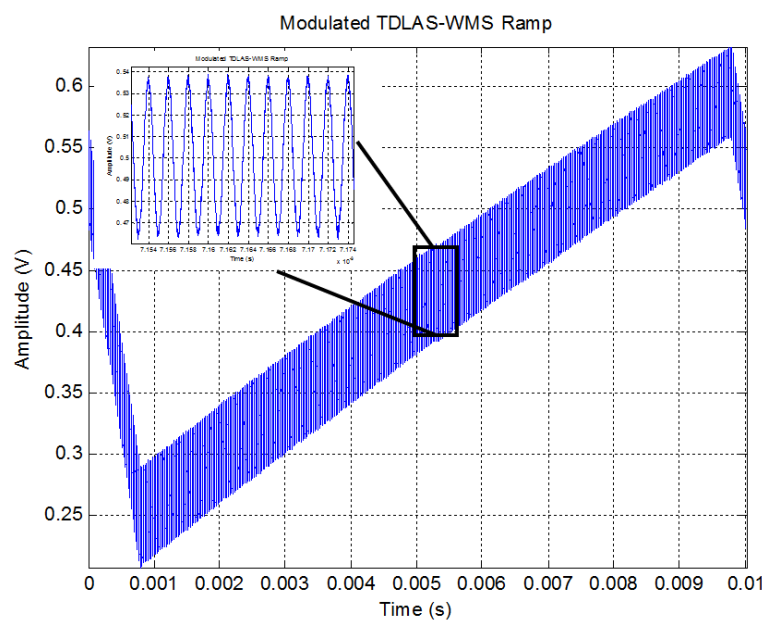

Fig. 1. Frequency-sweep across the full width of the gas absorption line
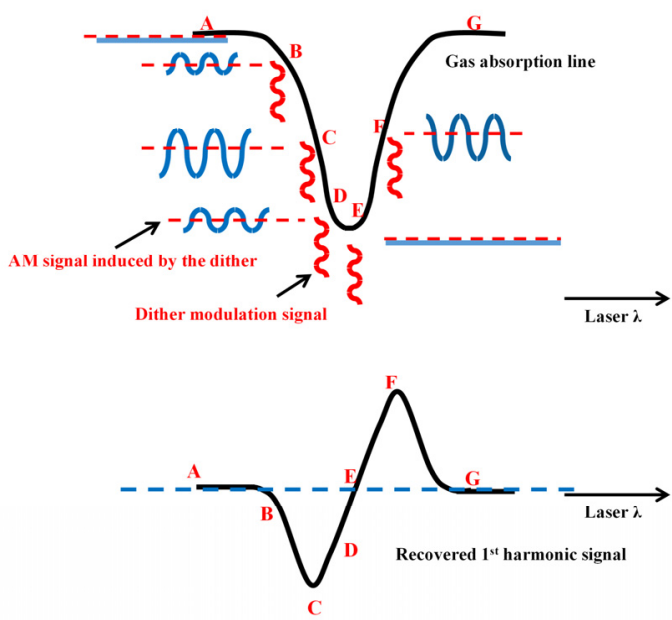

Fig. 2. Interaction between dither sine modulation and gas absorption line

$$
\alpha(\lambda)=\alpha(\lambda)+\alpha^{\prime}(\lambda)\left[\lambda-\lambda_{0}\right]+\alpha^{\prime \prime}(\lambda)\left[\lambda-\lambda_{0}\right]^{2}
$$

Then, substituting

$$
\left[\lambda-\lambda_{0}\right]=\delta \lambda \cos (\omega t-\psi)
$$

which represents the shift in laser peak wavelength due to the effect of the sinusoidal dither signal applied to the laser, $\psi$ being the phase shift between the intensity and the wavelength deviation, we obtain the following expression for the signal received by the photo-detector:

$$
\begin{aligned}
& I_{0} \approx[I+\Delta I \cos (\omega t)] \times\left[1-\alpha \cdot C l-\alpha^{\prime} \cdot C l \cdot \delta \lambda \cos (\omega t-\psi)+\right. \\
& \left.-\frac{1}{2} \alpha^{\prime \prime} \cdot C l \cdot \delta \lambda^{2} \cos ^{2}(\omega t-\psi)\right]
\end{aligned}
$$

The lock-in amplifier will then process the signal, isolating the $1 \mathrm{f}$ or $2 \mathrm{f}$ signal:

$$
\begin{aligned}
& I_{1 f}=\Delta I \cos (\omega t)-\Delta I \cdot \alpha \cdot C l \cdot \cos (\omega t)-I_{0} \alpha^{\prime} \cdot C l \cdot \delta \lambda \cos (\omega t-\psi) \\
& I_{2 f}=-\frac{1}{2} \Delta I \alpha^{\prime} C l \delta \lambda \cos (2 \omega t-\psi)-\frac{1}{4} I \alpha^{\prime \prime} C l \delta \lambda^{2} \cos 2(\omega t-\psi)
\end{aligned}
$$




\section{FPGA-based lock-in amplifier}

A lock-in amplifier measures an ac voltage and outputs a de signal proportional to the amplitude of the ac signal. It suppresses all the frequency contents outside the LPF pass band and outputs amplitude and phase information.

The basic principle of a digital lock-in amplifier is based on a quadrature demodulation scheme [12-14]. By multiplying the measured signal, amplitude $A$, with the two reference signals, amplitude $\mathrm{B}$, at the same angular frequency $\omega$ but with $90^{\circ}$ offset relative to each other, we get the in-phase and quadrature components. Each one will have a first term at the difference frequency, dc in this case, and a second term at the sum frequency $2 \mathrm{f}$. The in-phase $\left(\mathrm{V}_{\mathrm{I}}\right)$ and quadrature $\left(\mathrm{V}_{\mathrm{Q}}\right)$ signals are then filtered, expressed as

$$
\begin{aligned}
& V_{I}(n)=\sum_{n=1}^{N} A \cos (\omega n T+\theta) \cdot B \cos (\omega n T)=N \frac{A B}{2} \cos (\theta) \\
& V_{Q}(n)=\sum_{n=1}^{N} A \cos (\omega n T+\theta) \cdot B \sin (\omega n T)=N \frac{A B}{2} \sin (\theta)
\end{aligned}
$$

where $\mathrm{T}$ is the sampling time and $\mathrm{N}$ is the number of taps (12).

These results are the response of two PSD channels working synchronously and are proportional to the phase difference $\theta$ between the measured signal and the reference signal. Controlling the phase $\theta$ within the LIA, we can match the condition when the $1 \mathrm{f}$ (eqn 8) or $2 \mathrm{f}$ (eqn 9) signal is maximum, either for the $\mathrm{V}_{\mathrm{I}}$ or the $\mathrm{V}_{\mathrm{Q}}$ axis.

The FPGA module is implemented here using a Digilent Nexys 3 development board with a Xilinx Spartan6 FPGA. The resources available on this FPGA, in terms of number of fabric logic blocks, digital signal processing (DSP) slices, block RAM (BRAM) and $\mathrm{I} / \mathrm{O}$ paths, are adequate to implement our system. The development board provides standard interfaces, in particular for this design the USB interface is used to communicate with the PC. To implement the digital signal processing, a serial, 1MSPS, 12-bit precision ADC is used. Further, Xilinx provides state-of-the-art intellectual property (IP) blocks which are used throughout.

In order to implement successfully the digital lock-in detection scheme, the main requirements are frequencymatching between the measured and reference signal, and a constant phase difference over the acquisition period. For these reasons, the system provides user-controlled inputs for the phase and frequency of the reference signal.

In digital demodulation, a simple and low-resource method to filter the dc component can be implemented by a multiplyaccumulate (MAC) unit, using 1 DSP slice and a state machine controller which samples the accumulated value every $\mathrm{N}$ samples. The number of taps $\mathrm{N}$ defines the averaging period or measurement integration time of the DLIA. For testing and an adaptable instrument, $\mathrm{N}$ is a user defined input.

The proposed system in Fig. 3 samples the analogue signal at 1MSPS; sine and cosine reference signals are generated at the same frequency by a direct digital synthesizer (DDS) Xilinx IP core. The digitized measured signal is then sent to the dual channel PSD scheme and it is respectively multiplied by the cosine and sine signals. The MAC unit, made with a multiplier and an adder in cascade, operates by multiplying the two signals sample-by-sample and accumulating the results. A sampler then extracts the accumulated results after $\mathrm{N}$ iterations and the MAC unit is reset for the next accumulation.

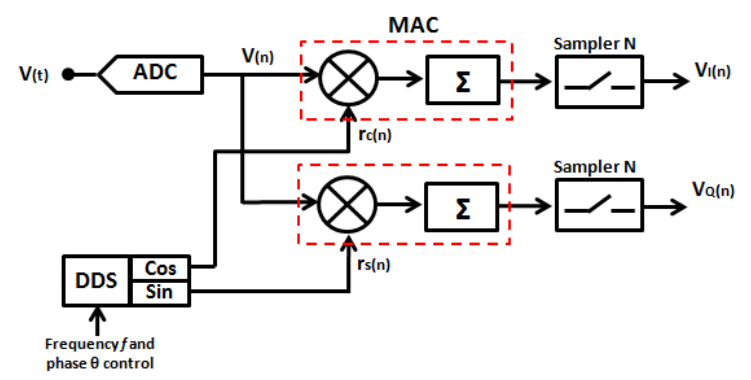

Fig. 3. The proposed implementation of a FPGA-based lock-in amplifier, where the filtering stage is implemented by using a MAC unit

The user defines $\mathrm{N}$, and thus the number of sine waves used by the averaging process. As the system operates in discrete time, the averaging period must contain an integer number of sine waves. Depending on the number of samples per sine period, the number of taps, $\mathrm{N}$, is defined as

$$
N=N u m_{-} \text {Samples } \cdot \mathrm{Num}_{-} \text {periods }
$$

The timing of these systems is dictated by the ADC sampling rate with each iteration within the number of taps being performed at this frequency. Assuming a $1 \mathrm{MHz}$ sampling rate $(1 \mu \mathrm{s})$, we can consider the averaging period $\mathrm{N}$ equivalent to a time constant of $\mathrm{N} \mu$ s. To improve the signal to noise ratio of the system, averaging over 20 dither periods is carrier out during the experimentation described below.

\section{TDLAS EXPERIMENTS AND RESULTS}

Fig.4 shows the basic experimental setup that was used to scan the $1997.2 \mathrm{~nm} \mathrm{CO}$ absorption line, in order to compare measurements from the FPGA-based DLIA and a Stanford Research System lock-in amplifier SR830. The diode-laser system consisted of a distributed feedback (DFB) diode laser manufactured by Eblana EP2000-DM-B. This was temperature and current controlled to operate in the $1997 \mathrm{~nm}$ region. A ramp $(5 \mathrm{~Hz})$ and sinusoidal $(25-50 \mathrm{kHz})$ current modulation were applied, sweeping and modulating the laser, to scan the range $1996.8-1997.6 \mathrm{~nm}$. The triggersynchronization signal from a function generator was sent to the lock-in amplifier to synchronize its reference signal.

Measurements were performed with sinusoidal modulation at $25 \mathrm{kHz}$ and $50 \mathrm{kHz}$; in both cases, the time constant for the FPGA DLIA acquisition was kept the same as for the SR830 LIA. For this purpose, the firmware programmed into the FPGA-DLIA is able to vary its accumulation time by changing the number of sine periods used within the averaging period. 


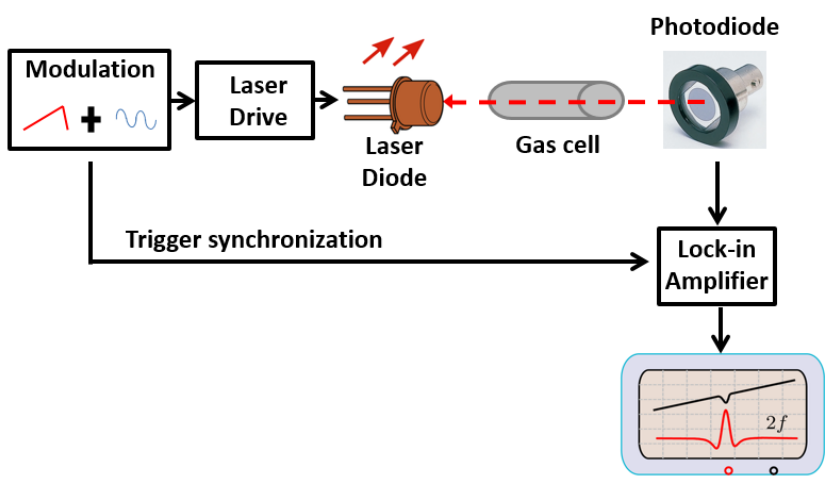

Fig. 4. Experimental digital TDLAS setup

The DFB laser output was coupled to fiber and then was coupled with a collimating lens into free space for the cell measurements. The gas cell is $5.5 \mathrm{~cm}$ long, with a $\mathrm{CO}_{2}$ gas concentration of $100 \%$, at a pressure of $\sim 1$ bar and temperature $15^{\circ} \mathrm{C}$. A photodiode detected the absorbed intensity and sent the signal to the SR830 LIA and to the FPGA-DLIA, which performed the $1 \mathrm{f}$ and $2 \mathrm{f}$ detection, and the outputs were captured using an oscilloscope and a PC, respectively. By adjusting the lock-in detection phase, while viewing the signal output and applying the criteria of achieving maximum signal strength and optimum symmetry, we were able to recover the first and second harmonics.
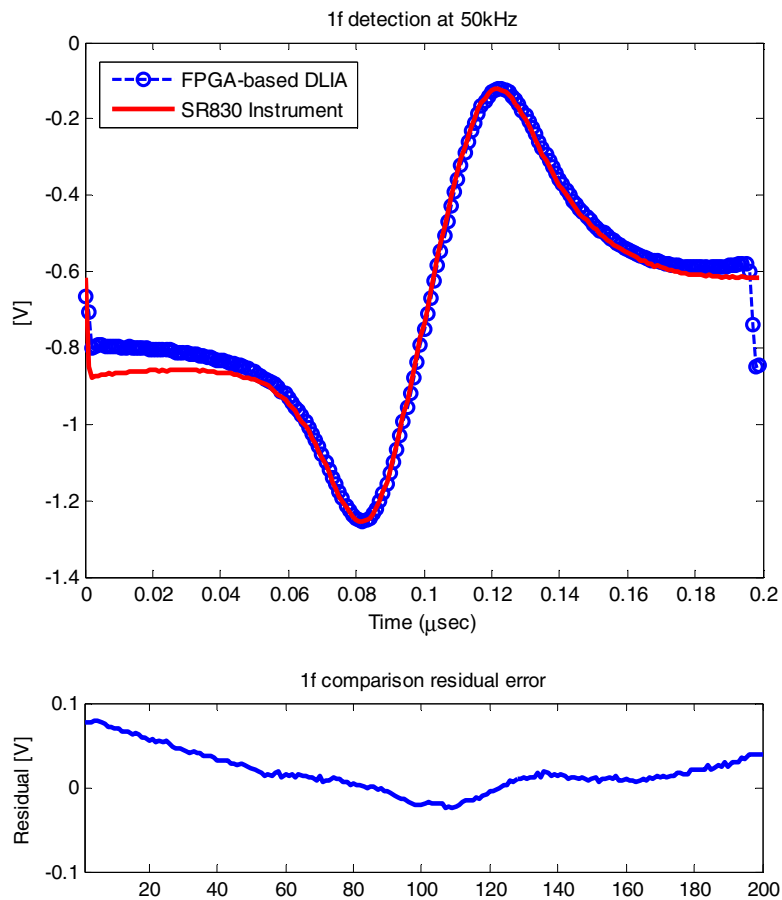

Fig. 5. First harmonic detection at $50 \mathrm{kHz}$. Comparison between FPGAbased digital lock-in and commercial lock-in amplifier (top) and residual error (bottom). CO2 - gas concentration: 100\%, gas pressure: 1000 mbar, gas temperature: $15^{\circ} \mathrm{C}$. Cell path-length $5,5 \mathrm{~cm}$.

Fig. 5 shows the comparison of $1 \mathrm{f}$ detection, modulating the laser with a $5 \mathrm{~Hz}$ ramp and $50 \mathrm{kHz}$ sine signals.
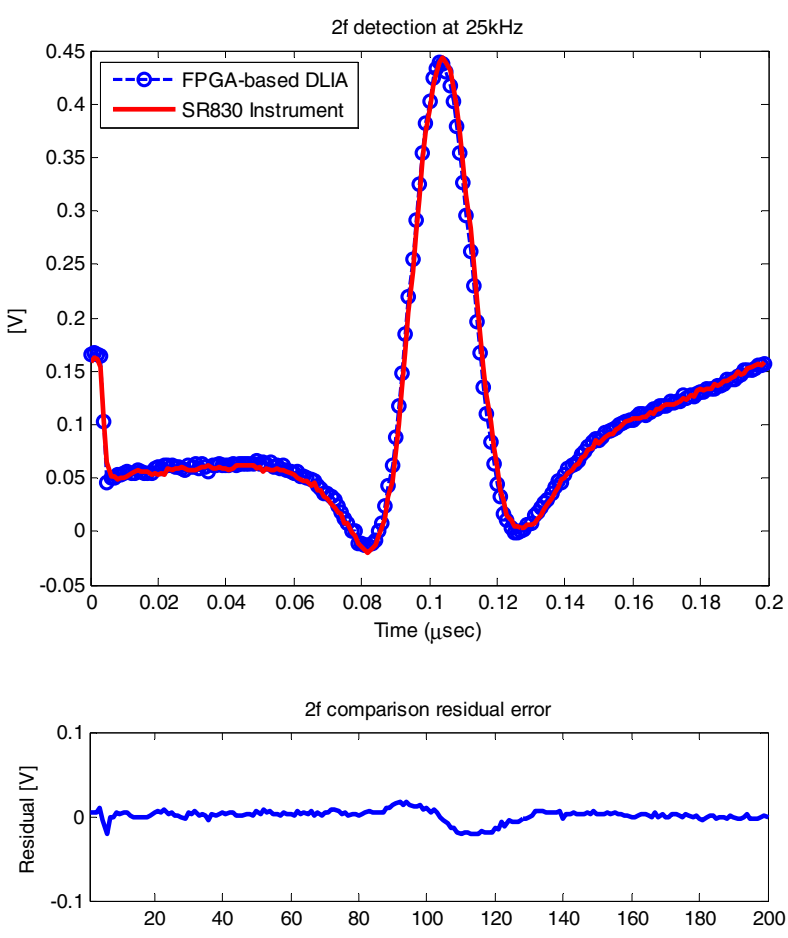

Fig. 6. Second harmonic detection at $25 \mathrm{kHz}$. Comparison between FPGA-based digital lock-in and commercial lock-in amplifier (top) and residual error (bottom). $\mathrm{CO} 2$ - gas concentration: 100\%, gas pressure: 1000 mbar, gas temperature: $15^{\circ} \mathrm{C}$. Cell path-length $5,5 \mathrm{~cm}$.

The second harmonic detection is carried out modulating the laser with $5 \mathrm{~Hz}$ ramp and $25 \mathrm{kHz}$ sine current signals. To perform $2 \mathrm{f}$ demodulation, the lock-in amplifier reference signals were tuned at $50 \mathrm{kHz}$, to double the frequency of the first harmonic, i.e. the laser intensity modulating signal. Fig. 6 shows $2 \mathrm{f}$ detection, comparing the acquisitions from the two different lock-in amplifiers.

From these preliminary results, we observe good matching between the detection performed with the FPGA-based DLIA and the conventional instrument SR830. There are however some differences, as can be observed on the residual plots, between the two LIAs. We expect the SR830 to have a much narrower filter, a higher sampling rate and bit resolution. Further improvements to these preliminary FPGA-based results would be achieved by using a non-square FIR filter, and moving to higher sampling rate and bit resolution.

\section{CONCLUSION}

A digital TDLAS system based on FPGA technology has been proposed and experimentally demonstrated, implementing the WMS $1 \mathrm{f}$ and $2 \mathrm{f}$ methods, to measure a narrow $\mathrm{CO} 2$ absorption feature. Its measurement results compare well with a commercially available bulk Stanford Research System SR830 lock-in amplifier instrument. 
These results validate the feasibility of the proposed system to exploit the properties of FPGA platforms to implement low-profile, low-weight and low-cost digital lockin amplifiers. The availability of resources in the FPGA provides scope for further development, and for extension of this system to a multi-channel TDLAS system. Thus, the method described here will enable applications that require a large number of measurement channels, notably for Chemical Species Tomography (CST). In particular, it facilitates the development of a 126-channel CST system for the FLITES project, as discussed in [10].

\section{Acknowledgment}

We are pleased to acknowledge the many helpful discussions we have enjoyed with our colleagues in the FLITES consortium and Yunjie Yang.

\section{References}

[1] M.E. Webber, S. Kim, S.T. Sanders, D.S. Baer, R.K. Handson, and Y. Ikeda "In situ combustion measurements of $\mathrm{CO} 2$ by use of a distributedfeedback diode-laser sensor near 2.0 um," Applied Optics, vol.40, no.6, 2001, 821-828.

[2] Wright $\mathrm{P}$, et al., "High-speed Chemical Species Tomography in a multicylinder automotive engine", Chem. Eng. J. vol. 158, no. 1, 2010 2-10.

[3] G.B. Rieker, J.B. Jeffries, and R.K. Hanson, "Calibration-free wavelength-modulation spectroscopy for measurements of gas temperature and concentration in harsh environments," Applied Optics, vol.48, no.29, 2009, 5546-5560.

[4] L. Mei, and S. Svanberg, "Wavelength modulation spectroscopy digital detection of gas absorption harmonics based on Fourier analysis," Applied Optics, vol. 54, no. 9, 2015, 2234-2243.
[5] K. Duffin, A.J. McGettrick, W. Johnstone, G. Stewart, and D.G. Moodie, "Tunable diode-laser spectroscopy with wavelength modulation: a calibration-free approach to the recovery of absolute gas absorption line shapes," Journal of Lightwave Technology, vol. 25, no. 10, 2007, 3114-3125.

[6] M. Andersson, L. Persson, T. Svensson, and S. Svanberg, "Flexible lock-in detection system based on synchronized computer plug-in boards applied in sensitive gas spectroscopy," Review of Scientific Instruments, vol.78, 113107 (7pp), 2007.

[7] S.J. Lascos, and D.T. Cassidy, "Multichannel digital phase sensitive detection using a field programmable gate array development platform," Review of Scientific Instruments, vol.79, 074702 (7pp), 2008.

[8] S.G. Castillo, and K.B. Ozanyan, "Field-programmable data acquisition and processing channel for optical tomography systems," Review of Scientific Instruments, vol.76, 095109 (6pp), 2005.

[9] J.R.P. Bain, M. Lengden, I. Armstrong, G.Stewart and W. Johnstone, "Development of tunable diode laser spectroscopy instrumentation for gas and species measurements in harsh environments," Applied Industrial Optics: Spectroscopy, Imaging and Metrology, Arlington, Virginia, USA, 2013.

[10] P. Wright, E. Fisher and H. McCann, "Optical design considerations for chemical species tomography in a jet exhaust plume," Laser Applications to Chemical, Security and Environmental Analysis, Seattle, Washington, USA, 2014.

[11] P. Wright, et al., "Progress towards Non-Intrusive Optical Measurement of Gas Turbine Exhaust Species Distributions," IEEE Aerospace Conference, Big Sky, Montana, USA, 2015.

[12] J.J. Vandenbussche, J. Peuteman, and P. Lee, "Development of a lowcost accurate phase measurement system," $1^{\text {st }}$ International Conference on Information and Communication Technologies Innovations and Applications, ICTIA, Sousse, Tunisia, 2014.

[13] H. Wang, Z. Cui, Y. Xu, L. Zhang, and Y. He, "Digital signal processing in electrical capacitance tomography," IEEE Asia Pacific Conference on Circuits and Systems, APCCAS, Macau, China, 2008.

[14] H. Zhou, L. Xu, Z. Cao, and C. Xu, "An alternative digital multiplication demodulation method for electrical capacitance tomography," IEEE International Instrumentation and Measurement Technology Conference, I2MTC, Graz, Austria, 2012. 